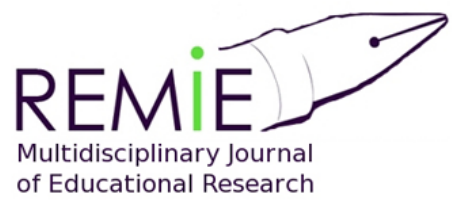

Instructions for authors, subscriptions and further details:

http://remie.hipatiapress.com

\title{
Las Urgencias Pedagógicas en la Sociedad del Aprendizaje y el Conocimiento. Un Estudio para la Reflexión sobre la Calidad en el Nuevo Modelo Educativo
}

Víctor Valdés ${ }^{1}$, Prudencia Gutiérrez-Esteban ${ }^{1}$

1) Universidad de Extremadura. España

Date of publication: February $15^{\text {th }}, 2018$

Edition period: February 2018-May 2018

To cite this article: Valdés, V. y Gutiérrez, P. (2018). Las Urgencias Pedagógicas en la sociedad del aprendizaje y el conocimiento. Un estudio para la reflexión sobre la calidad en el nuevo modelo educativo.

Multidisciplinary Journal of Educational Research, Vol (8), 1-28. doi:

10.17583/remie.2018.3199

To link this article: http://dx.doi.org/10.17583/remie.2018.3199

\section{PLEASE SCROLL DOWN FOR ARTICLE}

The terms and conditions of use are related to the Open Journal System and to Creative Commons Attribution License (CC-BY). 
REMIE - Multidisciplinary Journal of Educational Research Vol. 8 No. 1 February 2018

\section{The Pedagogical Priorities in the Learning and Knowledge Society. An Study for the Reflection on the Quality in the New Educational Model}

Víctor Valdés

University of Extremadura
Prudencia Gutiérrez-Esteban

University of Extremadura

(Received: 18 January 2018; Accepted: 10 February 2018; Published: 15 February 2018)

\section{Abstract}

The constant transformation that involves society requires a reflection about the educative sense and the detection of the Pedagogical Priorities in the educational context. This article analyzes the perspective of the university teachers and pupils about these questions. There were found evidences of the need of including or reinforcing aspects such as Citizenship Education, Emotional Education, as well as development of digital competences. The article is intended to demonstrate how the new social scenario is changing the educative model toward other quality standards linked with competences and abilities for the social development of people.

Keywords: aims of education, knowledge society, learning context, quality of education 
REMIE - Multidisciplinary Journal of Educational Research Vol. 8, No. 1 February 2018

\section{Las Urgencias Pedagógicas en la Sociedad del Aprendizaje y el Conocimiento. Un Estudio para la Reflexión sobre la Calidad en el Nuevo Modelo Educativo}

Víctor Valdés

Universidad de Extremadura
Prudencia Gutiérrez-Esteban

Universidad de Extremadura

(Recibido: 18 enero 2018; Aceptado: 10 febrero 2018; Publicado: 15 Febrero 2018)

\section{Resumen}

La constante transformación que envuelve a la sociedad demanda una reflexión acerca del sentido de la Educación y la detección de las Urgencias Pedagógicas latentes en el contexto educativo. El artículo analiza la visión del profesorado y el alumnado universitario en relación con estas cuestiones. Como resultado, se observan indicios de una necesidad de incluir o reforzar aspectos como la Ciudadanía, la Educación Emocional o las Competencias Digitales. De esta manera, se evidencia cómo la nueva realidad está cambiando el modelo educativo hacia otros estándares de calidad ligados a competencias y habilidades que permitan a las personas desarrollarse en sociedad.

Palabras clave: fines de la educación, sociedad del conocimiento, contexto de aprendizaje, calidad de la educación 


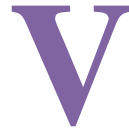

ivimos en una sociedad en constante transformación, lo que ha supuesto una actualización constante de los conocimientos y habilidades que poseemos. Esta realidad, a la que aluden Laal, Laal y Aliramaei (2014) o Turturean (2015), ha hecho que resurjan conceptos como el de formación permanente y formación a lo largo de la vida que, como señala Tünnermann (2010), aparecieron referidos a la educación para adultos pero que a finales del siglo $\mathrm{XX}$ volvieron a resurgir, siendo quizá el suceso más importante ocurrido en educación en la segunda mitad del siglo. Sobre esto, el Consejo de Universidades (2010) especifica que la formación permanente engloba los tres principales tipos de aprendizajes: el aprendizaje formal, no formal e informal/experiencial. Este último, está siendo cada vez más relevante y debe ser tenido en cuenta como un elemento vital de la educación tal y como sugieren Rimbau, Delgado y Rifá (2008) o Selwyn (2007). Los datos expuestos por Attwell (2007), contabilizan el aprendizaje informal como el $80 \%$ del conocimiento que posee una persona. Esta concepción del aprendizaje nos lleva a preguntarnos por la configuración de la educación del futuro, donde Moral (2008) considera imprescindible preparar al alumnado para que pueda vivir en la Sociedad del Aprendizaje y el Conocimiento, es decir, motivar la curiosidad por continuar aprendiendo y proporcionarles herramientas para que puedan aprender por sí mismos.

\section{La Sociedad Del Aprendizaje y El Conocimiento}

Cantón (2001, p. 202) determina que "la educación siempre será lo que sea la sociedad en la que se desenvuelve". Kornienko (2015) y Karpov (2017), entre otros, argumentan que esta nueva sociedad se basa en el conocimiento como un factor altamente importante en contextos sociales, políticos y económicos, dado que vivimos un momento donde las informaciones se han multiplicado gracias al avance de las tecnologías (Resende, 2013; Sevillano, 2005), permitiendo una distribución masiva del conocimiento, entrando de este modo en una concepción de la sociedad mundializada y globalizada a la que también alude Biesta (2014).

Para Tien (2013), Ricaurte (2016) o Zambrano Farías (2017), la sociedad del aprendizaje y el conocimiento también se caracteriza por una 
concepción del aprendizaje a lo largo de la vida (Areekul, Ratana-Ubol, \& Kimpee, 2015), entendiendo la educación como una actividad abierta que no puede limitarse a espacios cerrados, que reconoce las diferencias individuales de cada persona a la hora de adquirir el aprendizaje e intenta dar respuesta a cada una de ellas, cuenta con una estructura física y cada vez más, virtual, para dar respuesta a las demandas educativas y apuesta por la innovación como motor de evolución.

Del mismo modo, Area y Pessoa (2012), Negre, Marín y Pérez (2013), Bhatt (2016) recalcan las nuevas capacidades demandadas por esta sociedad, que van desde la capacidad para adaptarse a los cambios hasta la capacidad para manejar, gestionar y curar la información (Ponerulappan y Thilagavathy, 2014), es decir, ser capaz de clasificar y seleccionar entre el grueso del contenido existente. La unificación de todas las capacidades podría denominarse la Competencia Informacional, que es definida por Area (2010, p.52) como "las habilidades, conocimientos, disposiciones y conductas que capacitan a los individuos para reconocer cuándo necesitan información, dónde localizarla, cómo evaluarla y darle un uso adecuado de acuerdo con el problema que se les plantea".

Aunque aún estamos lejos de algunos de los patrones que caracterizan esta educación, sí se están dando pequeños pero continuados pasos que conllevan un replanteamiento del sentido de la educación y la introducción de una serie de elementos que urgen ser incluidos en nuestra realidad educativa.

\section{Urgencias Pedagógicas}

De acuerdo con este contexto social, la Educación deber dar una respuesta acorde con los tiempos, en consonancia con las características de la Sociedad del Aprendizaje y el Conocimiento. Se trata de lo que hemos denominado a partir de estudios previos como el de Contreras y Pérez de Lara (2010), Urgencias Pedagógicas. Así, consideramos "Urgencia Pedagógica" a aquellos aspectos que el profesorado debería abordar en las aulas con cierta premura. Tras el análisis de la literatura precedente, las Urgencias Pedagógicas se vertebran en torno a los siguientes ejes: 


\section{A. El Autoaprendizaje En La Educación No Formal E Informal}

Ya no existe un tiempo y lugar determinado para aprender, por lo que cada vez es más urgente una nueva cultura de aprendizaje que rompa con los métodos tradicionales que no están dando respuesta a la actual realidad educativa. Cómo señalan Gutiérrez Esteban y Mikiewicz (2012), este panorama social, económico y cultural ha supuesto cambios en el paso de un paradigma formal de educación hacia un modelo Do-It-Yourself (DIY) (Kafai \& Peppler, 2011) en el que el aprendizaje traspasa los muros de la escuela y es más eficaz cuando el alumnado consigue desarrollar el aprendizaje en los dos contextos, tanto en el formal como en el informal (Hall, 2009).

Así, el aprendizaje se convierte gradualmente en invisible, tal y como explican Cobo y Moravec (2010). Es decir, los jóvenes cada vez más aprenden alejados de los sistemas educativos formales gracias a las nuevas vías de formación y aprendizaje. Además, este fenómeno se ve altamente facilitado por los recursos tecnológicos que ofrece la Web 2.0, dando lugar a los PLE (Personal Learning Environments) y los PLN (Personal Learning Networks). Dabbagh y Kitsantas (2012) y Gutiérrez y Becerra (2014) apuestan por la utilización de estos PLE para la unificación del aprendizaje formal y no formal mediante el fomento de comunidades virtuales de aprendizaje. Lo que hace que se favorezca el autoaprendizaje y a que el alumnado busque información en función de sus propios intereses, motivaciones, objetivos y expectativas (Rahimi, Van den Berg, y Veen, 2015).

\section{B. Educación Para La Ciudadanía}

Como apunta Gómez (2000) o Leite, Fernandes, y Marques da Silva (2013), trabajar la ciudadanía es un tema controvertido en nuestra sociedad, es un concepto ideológico, cultural, sujeto a perspectivas y diferentes visiones de la misma. Aun así, es importante educar en una serie de valores ciudadanos como pueden ser:

1. La necesidad de agentes sociales. La educación debe propiciar la actitud e identidad social de las personas. Para Vergara et al. (2008) 
o Fernández-Soria y Mayordomo (2014) debemos transmitir valores de justicia, libertad ciudadana, servicio social, compromiso, esfuerzo, generosidad, altruismo y participación democrática, entre otros.

2. El trabajo por la igualdad. Hoy en día, como recogen García, et al. (2010) o Anguita (2011), todavía aparecen con mucha frecuencia elementos sexistas en educación, como es el caso del uso generalizado de un lenguaje sexista o los materiales educativos y currículum androcéntrico (Cobo, 2008).

3. Educación para la resolución de conflictos. Normalmente, apunta Funes (2000), se entiende el conflicto como algo negativo, puesto que se asocia a la forma violenta en la que se suelen resolver. La forma positiva de trabajar el conflicto sería mediante la mejora de habilidades sociales concretas como la comunicación, que destacan Rodríguez Fuentes, et al. (2017) o la negociación y la mediación en las que De Lara (2008) incide en el plan de convivencia de su centro.

4. Educación para el desarrollo humano y sostenible. Este tipo de desarrollo busca lo que Rodríguez, Orozco y Larena (2011, p.130) describen como "una sociedad económicamente dotada, equitativamente distribuida, propietaria de un comercio justo, socialmente igualitaria, no consumista, altamente capacitada, poseedora de unos valores integralmente humanos, interculturalmente cosmopolita, humana y ecológica". Todo ello necesario para conseguir la relación armónica entre la humanidad y el medio natural que autores como Kim y Kim (2013) defienden.

La educación para la ciudadanía es un tema extenso que, tal y como indica García Raga y López Martín (2013), se ha convertido en un objetivo prioritario de todo proceso educativo. Se trata de alcanzar la denominada "cultura de convivencia" que ya es una realidad en casos como el que describe Molina Girón (2013) y del cual se podrían extraer muchas categorías. En este trabajo, se han destacado algunas de ellas por relevancia social quizás, pero no queriendo con ello establecer las fronteras del trabajo para la ciudadanía que debe seguir avanzando y transformándose conforme 
evoluciona la sociedad para atender a las nuevas demandas que puedan surgir (Palmer, 2017).

\section{Educación Emocional}

Las emociones son un aspecto importante de las personas, en cuanto a que determinan el estado de ánimo e influyen en nuestro comportamiento (Morilla-García, 2017). Por ese motivo es tan importante tener competencias emocionales que permitan gestionarlas. Cera (2012, pp. 3940) describe las competencias emocionales como:

Las capacidades relativas a la mejora personal como el autoconocimiento, la autoestima, el autocontrol o la motivación, la creatividad, la capacidad para el cambio o para la toma de decisiones; y de otra a aquellas relativas a las relaciones con los demás como la empatía, la capacidad para establecer una adecuada comunicación para trabajar en equipo, para resolver conflictos, para analizar las necesidades de la sociedad o los mercados, para ser líderes en diferentes situaciones.

Por el contrario, Goleman (1996) denomina la ausencia de dichas competencias como "analfabetismo emocional", es decir, la incapacidad para gestionar las emociones, siendo éste un problema igual de serio que un déficit en materias curriculares. Las personas denominadas analfabetas emocionales presentan dificultades para desarrollar las habilidades emocionales básicas: reconocer, aceptar, gestionar, expresar y crear emociones.

Como se puede apreciar en la tabla, las urgencias pedagógicas pueden surgir de diferentes naturalezas. Así, detectamos, por ejemplo, la demanda de una serie de competencias, que hasta ahora la sociedad no había reclamado al ámbito educativo, pero que empiezan a ser indispensables. Se puede observar el intento por impregnar la educación de un carácter social, que atienda a la persona íntegramente en todas sus dimensiones, confiando en que este cambio de orientación consiga mejorar la sociedad. Partiendo de este contexto, Masini (2013) nos invita a crear un nuevo diseño de Educación para un futuro que cada vez está más presente y el cual debe ser tenido en cuenta. En esta nueva realidad no se puede racionalizar todo, las ideas estáticas producen una sensación de comodidad que impide adentrarse 


\section{Valdés \& Gutiérrez-Urgencias Pedagógicas}

en aquello que todavía se desconoce. Por ello, debe existir una apertura a lo inesperado, a lo desconocido.

Tabla 1:

Relación de Urgencias Pedagógicas detectadas en la revisión de la literatura

\begin{tabular}{ll}
$\begin{array}{l}\text { Autoaprendizaje y } \\
\text { educación no formal }\end{array}$ & $\begin{array}{l}\text { trabajo con PLE } \\
\text { capacitar gestores de conocimiento }\end{array}$ \\
\hline $\begin{array}{l}\text { Educación para la } \\
\text { ciudadanía }\end{array}$ & $\begin{array}{l}\text { capacitar agentes sociales } \\
\text { trabajar para la igualdad } \\
\text { educación para la resolución de conflictos } \\
\text { educación para el desarrollo humano y sostenible }\end{array}$ \\
\hline & competencias emocionales \\
Educación emocional & inteligencia emocional \\
& alfabetización emocional
\end{tabular}

\section{Método}

Este estudio realizado entre los meses de enero y septiembre de 2015, nace con el objetivo de reflexionar sobre el sentido de la Educación en la Sociedad del Aprendizaje y el Conocimiento e identificar las Urgencias Pedagógicas que se desprenden del contexto social y tecnológico. Para alcanzar dicho objetivo se plantean dos preguntas de investigación: ¿Qué sentido se le da actualmente a la educación en el contexto de la sociedad del aprendizaje y el conocimiento? y ¿Cuáles son las principales urgencias pedagógicas que se detectan desde la comunidad educativa universitaria?

La muestra está compuesta por profesores y profesoras universitarias y estudiantes de los Grados de Educación Infantil y Primaria de la Facultad de Educación de la Universidad de Extremadura. Para la selección de la muestra se ha seguido un muestro no probabilístico, en el grupo de estudiantes se ha desarrollado un muestreo por conveniencia; es decir, se ha seleccionado la muestra por la facilidad de acceso. En el caso del profesorado universitario en cambio, se ha utilizado un muestreo intencional; es decir, se han seleccionado a las personas participantes, por 
su idoneidad para la investigación. Las personas seleccionadas forman parte del profesorado de la misma facultad que imparte docencia en los grados anteriormente mencionados.

\section{Tabla 2:}

Descripción de participantes en el estudio

\begin{tabular}{|c|c|c|c|c|}
\hline $\begin{array}{l}\mathrm{N}^{\mathrm{o}} \text { total de } \\
\text { participantes }\end{array}$ & $\begin{array}{l}\text { Perfil } \\
\text { participantes }\end{array}$ & $\begin{array}{l}\mathrm{N}^{\circ} \text { de participantes } \\
\text { por perfil }\end{array}$ & Edad & Sexo \\
\hline \multirow{4}{*}{19} & Profesorado & \multirow{2}{*}{1} & \multirow{2}{*}{$\begin{array}{l}39 \text { a } \\
62 \\
\text { años }\end{array}$} & $5 \mathrm{H}$ \\
\hline & $\begin{array}{l}\text { Protesorado } \\
\text { Universitario }\end{array}$ & & & $6 \mathrm{M}$ \\
\hline & \multirow{2}{*}{$\begin{array}{l}\text { Alumnado } \\
\text { Universitario }\end{array}$} & \multirow[b]{2}{*}{8} & \multirow{2}{*}{$\begin{array}{l}21 \text { a } \\
26 \\
\text { años }\end{array}$} & $2 \mathrm{H}$ \\
\hline & & & & $6 \mathrm{M}$ \\
\hline
\end{tabular}

Los instrumentos elegidos para la recogida de datos en la investigación han sido la entrevista personal semiestructurada y el grupo de discusión. De acuerdo con Strauss y Corbin (2002) se escogen dos instrumentos para la recogida de datos con el fin de alcanzar mayor objetividad, necesaria para poder desarrollar un estudio imparcial y preciso. Por este mismo motivo, al elegir la muestra, se ha contado tanto con profesorado universitario como con alumnado, con la intención de representar todas las perspectivas posibles.

Profundizando en los instrumentos, se ha optado por utilizar el grupo de discusión con el alumnado ya que este instrumento favorece la participación de las personas implicadas puesto que se ven reforzadas por el diálogo que se genera en la interacción con el resto de participantes. En cambio, se ha empleado la entrevista con el profesorado dado que permite recoger en profundidad las vivencias y perspectivas, así como la dilatada experiencia del profesorado.

\section{Análisis de Datos}

En este caso, para la categorización de los datos recabados se utilizó un criterio temático de acuerdo con la propuesta de Yuste (2012), de manera que se identifican segmentos/referencias que hablan de un mismo tema a lo 


\section{Valdés \& Gutiérrez-Urgencias Pedagógicas}

largo de las entrevistas y grupos de discusión. Asimismo, se agrupan los segmentos, los cuales se elevan en categorías y éstas en dimensiones, siempre en función de la similitud temática. La distribución de los segmentos/referencias en las diferentes categorías, así como el posterior análisis, se ha llevado a cabo mediante la utilización del software de análisis cualitativo NVIVO 10 que permite fragmentar la información en nodos/categorías. En la siguiente tabla se muestra el resultado del análisis.

Tabla 3:

Dimensiones, categorías y segmentos

\begin{tabular}{llll} 
Dimensiones & Categorías & Segmentos \\
\hline $\begin{array}{l}\text { Sociedad del } \\
\text { aprendizaje y el } \\
\text { conocimiento }\end{array}$ & Sentido de la educación & 46 segmentos \\
\hline $\begin{array}{l}\text { Urgencias } \\
\text { pedagógicas }\end{array}$ & $\begin{array}{l}\text { Autoaprendizaje y educación no } \\
\text { formal }\end{array}$ & $\begin{array}{l}\text { Educación para la ciudadanía } \\
\text { Educación emocional }\end{array}$ & 63 segmentos \\
&
\end{tabular}

\section{Resultados}

Para una mejor comprensión de los resultados se han organizado siguiendo las preguntas de investigación planteadas al inicio de este estudio.

\section{¿Qué Sentido Se Le Da Actualmente A La Educación En El Contexto De La Sociedad Del Aprendizaje y El Conocimiento?}

Sobre el sentido de la educación se han encontrado un total 46 segmentos/referencias de la información recogida de las entrevistas y grupos de discusión. En la siguiente nube de palabras generada desde NVIVO 10, se aprecian los términos más frecuentes que han aparecido al hablar del sentido de la educación. 


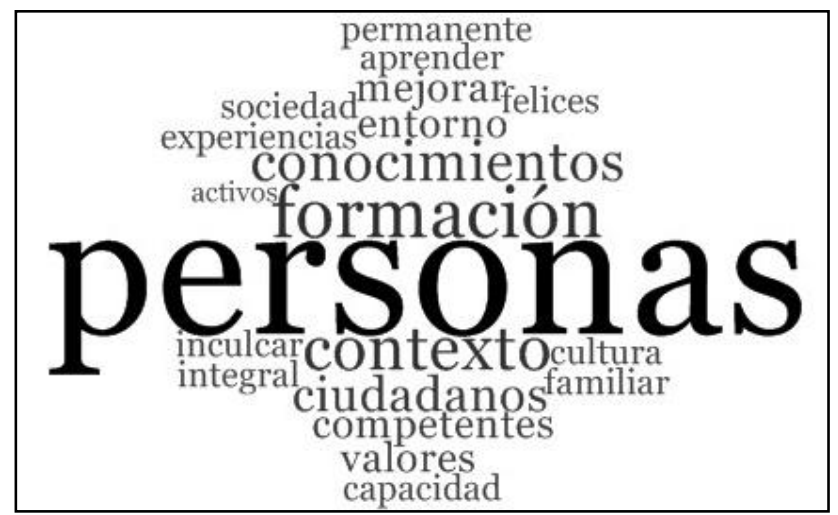

Figura 1. Nube de palabras del Sentido de la Educación.

El total de personas participantes del estudio reconoce que para ellas el fin último de la educación es formar buenas personas o personas felices. En cambio, no hay evidencias de posicionamientos ligados a la transmisión de conocimientos o la selección de los sujetos más capacitados. Para profundizar un poco más, en la siguiente tabla se muestran tres segmentos con las reflexiones más representativas acerca del sentido de la educación.

Los tres segmentos reflejan el conjunto de respuestas obtenidas a lo largo del estudio. En el tercer segmento, a pesar de ser el más corto, se recoge la idea que subyace al resto de reflexiones, "se trata de una cuestión más moral o más ética que técnica". Así, se denota una evolución desde el perfil asistencial de los inicios de la educación, hasta éste nuevo enfoque donde se demanda la atención a una formación moral, ciudadana, que prepare para el futuro, para el dominio de las tecnologías y desarrolle capacidades deportivas, artísticas y culturales, entre otras.

En el segundo segmento, "no se entiende la educación por cuatro reglas para saber comportarse", se percibe la distancia entre la concepción de la educación como instructora de las principales áreas disciplinares y la educación entendida como una búsqueda del crecimiento personal, la libertad, la felicidad, o la formación integral de la persona. 
Tabla 4

Ejemplos de segmentos referidos a la categoría de Sentido de la Educación.

\begin{tabular}{|c|c|c|c|}
\hline Categoría & $\begin{array}{c}\mathrm{N}^{\circ} \mathrm{de} \\
\text { referencias }\end{array}$ & Participantes & Segmento \\
\hline \multirow{3}{*}{$\begin{array}{l}\text { Sentido de } \\
\quad \text { la } \\
\text { educación }\end{array}$} & \multirow{3}{*}{46} & Prof1 & $\begin{array}{l}\text { "Yo creo que el sentido fundamental es } \\
\text { hacernos libres y hacernos personas... que } \\
\text { seamos ciudadanos primero, felices y } \\
\text { después ciudadanos activos que } \\
\text { contribuyan positivamente a su sociedad." }\end{array}$ \\
\hline & & Prof5 & $\begin{array}{l}\text { "Es la formación integral de una persona, } \\
\text { no entendiendo por educación solo cuatro } \\
\text { reglas para la manera de comportarse, si } \\
\text { no su formación, sus experiencias, todo." }\end{array}$ \\
\hline & & Prof3 & $\begin{array}{l}\text { "La educación es mejorar a las personas, } \\
\text { eso es lo importante, es una cuestión más } \\
\text { digamos moral o más ética que técnica." }\end{array}$ \\
\hline
\end{tabular}

\section{¿Cuáles Son Las Principales Urgencias Pedagógicas Que Se Detectan Internamente En La Formación Inicial Del Profesorado?}

En cuanto a las Urgencias Pedagógicas, se trata de la categoría que más segmentos contiene, (63 segmentos) en las cuales aparecen todo tipo de necesidades educativas detectadas por el profesorado y el alumnado universitario participante en el estudio. A continuación, se muestra de nuevo una nube de palabras con los términos más frecuentes encontrados en las transcripciones. Posteriormente, se exponen las principales Urgencias Pedagógicas detectadas.

Las palabras más frecuentes son competencia y competencias (Figura 2). Esto es debido a que normalmente han aparecido junto a otros términos, como competencia digital, competencia comunicativa o competencia emocional. La aparición reiterada refleja la importancia que se le está otorgando, desde hace unos años, al trabajo por competencias; es decir, el saber hacer por encima de los contenidos, los cuales también aparecen reflejados en los segmentos analizados. Se trata de ser capaz de utilizar lo aprendido fuera del contexto educativo reordenando y modificando los 
conocimientos y saberes para poder hacerlo. A continuación, se desarrollan las subcategorías que cuentan con mayor número de segmentos.

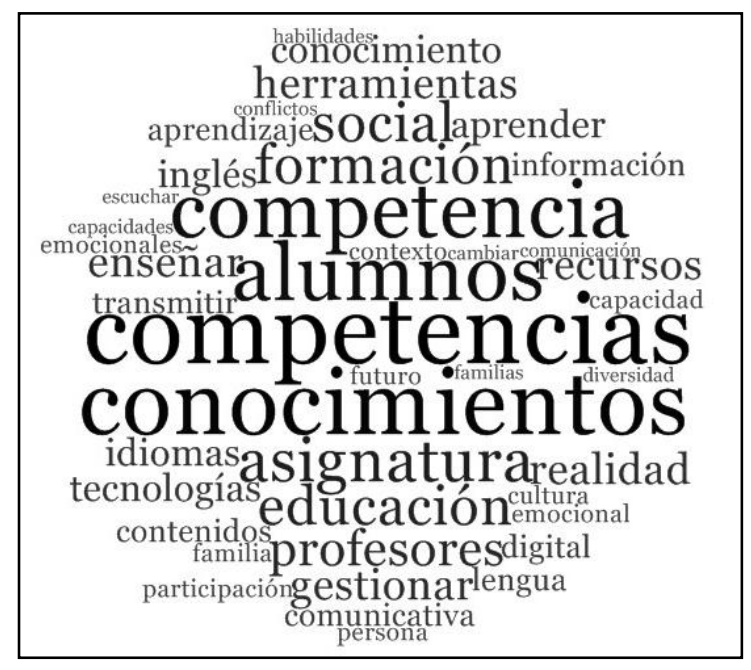

Figura 2. Nube de palabras de las Urgencias Pedagógicas.

I. Autoaprendizaje en educación no formal. En la Sociedad del Conocimiento, las tecnologías e Internet juegan un papel fundamental. Como recogen los segmentos anteriores, se debe ampliar la formación en competencias digitales y ofrecer una formación en el uso educativo de los recursos tecnológicos. De la misma forma, hay que formar al profesorado como gestores de contenidos para que sepan seleccionar información, mantener los contenidos actualizados y sepan enseñar a seleccionar información dentro de la gran fuente que es Internet. La mayor parte de la población es incapaz de seleccionar entre tanta información aquella que les resulta útil y que a su vez cuenta con unos mínimos de calidad. Ante esta situación ser capaz de llevar a cabo una curación de contenidos, es decir, clasificar el contenido existente para proporcionar mayor calidad a los usuarios se convierte en una tarea prioritaria. 


\section{Valdés \& Gutiérrez-Urgencias Pedagógicas}

Tabla 5

Selección de segmentos referidos a la categoría de Urgencias Pedagógicas y Autoaprendizaje en educación no formal.

Categoría Subcategoría $\quad \mathrm{N}^{\circ}$ de Participante Segmento referencias

\begin{tabular}{|c|c|c|c|}
\hline & & Prof9 & $\begin{array}{l}\text { “...habría que hacer un } \\
\text { hincapié tremendo y pasar de } \\
\text { lo que se enseña actualmente, } \\
\text { que está muy bien, pero en la } \\
\text { universidad no deberíamos } \\
\text { estar para enseñar a hacer un } \\
\text { blog, por ejemplo, porque eso } \\
\text { es tan tremendamente básico } \\
\text { que eso no puede ser de } \\
\text { ninguna manera”, }\end{array}$ \\
\hline $\begin{array}{l}\text { Urgencias } \\
\text { pedagógicas }\end{array}$ & $\begin{array}{l}\text { Autoaprendizaje } \\
\text { en educación no } \\
\text { formal }\end{array}$ & Alum1 & $\begin{array}{l}\text { "Competencia digital } \\
\text { muchísima, porque ya } \\
\text { deberíamos estar actualizados } \\
\text { totalmente en ese aspecto y no } \\
\text { lo estamos. Por lo tanto, } \\
\text { cuando pasen unos años vamos } \\
\text { a estar por debajo de lo que los } \\
\text { niños van a saber." }\end{array}$ \\
\hline & & Prof10 & $\begin{array}{l}\text { "tenemos alumnos que cada } \\
\text { vez están más informados, a lo } \\
\text { mejor con una información } \\
\text { sesgada, porque no tienen } \\
\text { todavía la capacidad de abrir } \\
\text { el abanico y de percibir los } \\
\text { distintos puntos de vista de un } \\
\text { mismo tema, pero claro, } \\
\text { primero los alumnos van en } \\
\text { muchísimos aspectos por } \\
\text { delante de nosotros, en las } \\
\text { aulas está entrando la } \\
\text { tecnología y necesitamos } \\
\text { utilizarlas como instrumentos y } \\
\text { como herramientas." }\end{array}$ \\
\hline
\end{tabular}

II. Educación para la ciudadanía. Se aprecia como urgencia pedagógica el cuidado del planeta, el pensamiento crítico, la tolerancia o la sensibilidad hacia los principales problemas sociales que están aconteciendo por todo el mundo. Se entiende, por tanto, la necesidad de proporcionar una formación 
humana y social que desarrolle el crecimiento personal de los estudiantes y que pueden llevarse a la práctica a través de la transmisión de contenidos curriculares, en la relación entre estudiantes y profesorado, en la organización del aprendizaje, en la cultura institucional o mediante la implicación comunitaria de la comunidad educativa.

Tabla 6

Selección de segmentos referidos a la categoría de Urgencias Pedagógicas y educación para la ciudadanía.

\begin{tabular}{|c|c|c|c|c|}
\hline Categoría & Subcategoría & $\begin{array}{l}\mathrm{N}^{\circ} \mathrm{de} \\
\text { referencias }\end{array}$ & Participante & Segmento \\
\hline \multirow[b]{2}{*}{$\begin{array}{l}\text { Urgencias } \\
\text { pedagógicas }\end{array}$} & \multirow[b]{2}{*}{$\begin{array}{l}\text { Educación } \\
\text { para la } \\
\text { ciudadanía }\end{array}$} & \multirow[b]{2}{*}{19} & Prof3 & $\begin{array}{l}\text { "Aspectos sobre la formación como } \\
\text { persona de tus alumnos } \\
\text { posiblemente no va a cambiar } \\
\text { nunca. enseñar a un maestro a } \\
\text { cómo enseñar tolerancia en sus } \\
\text { clases o como enseñar } \\
\text { participación o preocupación } \\
\text { social, eso a lo mejor no va a } \\
\text { cambiar tanto como van a cambiar } \\
\text { otras cosas." }\end{array}$ \\
\hline & & & Prof3 & $\begin{array}{l}\text { "Aspectos como qué va a ser de } \\
\text { nuestro planeta o qué va ser de } \\
\text { nuestra sociedad mañana o qué } \\
\text { pasa con la gente que está llegando } \\
\text { de áfrica y que se están muriendo } \\
\text { en el mediterráneo, o qué pasa con } \\
\text { la gente que en los pueblos está } \\
\text { parada y está viviendo con } 400 \\
\text { euros al mes cinco personas, eso yo } \\
\text { creo que los maestros también } \\
\text { teníamos que ver algo, no podemos } \\
\text { ser... todo ese tipo de cosas, esa } \\
\text { sensibilidad hay que creársela a los } \\
\text { profesores." }\end{array}$ \\
\hline
\end{tabular}

\section{Educación emocional.}

La inteligencia emocional cada vez está siendo más importante en la formación de una persona. La gestión de las emociones permite alcanzar 


\section{Valdés \& Gutiérrez-Urgencias Pedagógicas}

una estabilidad emocional que es un factor muy importante para desarrollar cualquier proyecto vital. La identificación de las emociones y la capacidad de diferenciar entre sentimientos de distinto tipo es fundamental para poder hacer frente a otras competencias educativas desde un posicionamiento personal adecuado y seguro. Por eso, se revela como un elemento a tener muy en cuenta dentro de la educación, además los nuevos ritmos, horarios y estilos de vida, hacen que sea especialmente necesario.

A través de las reflexiones sobre el sentido de la educación y algunas de las propuestas y urgencias detectadas, se dejan entrever intenciones de poner en marcha una práctica educativa más social y más reflexiva, ligada a la realidad que nos rodea.

Tabla 7

Selección de segmentos referidos a la categoría de Urgencias Pedagógicas y educación emocional.

\begin{tabular}{|c|c|c|c|c|}
\hline Categoría & Subcategoría & $\begin{array}{l}\mathrm{N}^{\mathrm{o}} \mathrm{de} \\
\text { referencias }\end{array}$ & Participante & Segmento \\
\hline \multirow{3}{*}{$\begin{array}{l}\text { Urgencias } \\
\text { pedagógicas }\end{array}$} & \multirow{3}{*}{$\begin{array}{l}\text { Educación } \\
\text { emocional }\end{array}$} & \multirow{3}{*}{10} & alum2 & $\begin{array}{l}\text { "tú a un alumno tienes que } \\
\text { enseñarle a expresarse, a mostrar } \\
\text { sus emociones, con eso también } \\
\text { empatizan con los demás y los } \\
\text { convierten en personas } \\
\text { emocionales y capaces de } \\
\text { afrontar situaciones y tener } \\
\text { sociabilidad." }\end{array}$ \\
\hline & & & alum1 & $\begin{array}{l}\text { "desde los tres años que entra un } \\
\text { niño en el colegio, se pueden } \\
\text { hacer muchísimas cosas de } \\
\text { inteligencia emocional y de } \\
\text { educación emocional con ellos." }\end{array}$ \\
\hline & & & prof8 & $\begin{array}{l}\text { "competencias emocionales, } \\
\text { tienen que adquirir competencias } \\
\text { emocionales y por supuesto de } \\
\text { conocimiento, tiene que saber lo } \\
\text { máximo posible porque tiene que } \\
\text { transmitir y saber cómo lo } \\
\text { transmiten, pero sobretodo } \\
\text { competencias emocionales." }\end{array}$ \\
\hline
\end{tabular}




\section{Conclusiones}

A pesar de que el sentido de la educación nunca ha sido ni será estático, sino que cambia y evoluciona en concordancia con la sociedad, (FernándezSoria \& Mayordomo, 2014; Kartashova, 2015). Las valoraciones recogidas durante este trabajo acerca del sentido de la educación invitan a pensar que estamos más cerca de la educación que Delors (1996) planteó para el siglo XXI. Aun así, se percibe una disociación entre los planteamientos educativos y la realidad del sistema. Esto es debido principalmente por dos motivos, el incipiente cambio en la concepción del rol que ejercen, al pasar de ser ciudadanos a clientes, tal como alude Pérez Gómez (2000) y el uso de la educación como un instrumento del estado, sin responder a las principales necesidades sociales (Gimeno Sacristán, 1982). Responsable de esto es el contexto globalizado en el que nos situamos debido a la vigencia de una economía neoliberal. Esta concepción creada por el mercado ha llegado a estamentos que tradicionalmente habían gozado de un estatus público y homogéneo, como la sanidad o la educación. Esto supone la diferenciación y la desigualdad de la sociedad puesto que permite a las familias que gozan de mayor nivel económico ofrecer una mejor educación que la del resto de familias con menos recursos, (Gutiérrez Díez, 2015). Esta situación complica la consecución de un objetivo primordial en educación, que no es otro que el de conseguir que todas las generaciones puedan desarrollarse en igualdad de condiciones independientemente de la procedencia del alumnado. Para conseguir ese objetivo precisamos de un cambio en la concepción de la educación.

Como explica Gómez (2000), se trata de pasar de una escuela cuyo objetivo es la perpetuación del orden social existente, a una escuela que sirva de motor de cambio y que permita la igualdad de oportunidades. Como se refleja en las opiniones vertidas por el profesorado y el alumnado universitario participante en el estudio, podemos estar viviendo un cambio en Educación hacia una concepción de formación integral de la ciudadanía, enfatizando aspectos como la felicidad y la capacitación social de las personas. En este escenario, es necesario también saber contextualizar, ya no existen las verdades absolutas y en esta nueva realidad hay que hilar la inteligencia a las nuevas Urgencias Pedagógicas. Hay que aprender dentro 


\section{Valdés \& Gutiérrez-Urgencias Pedagógicas}

de la vida y no de manera aislada, hay que dejarse llevar por la curiosidad. Debe entenderse la vida como un proceso constante de orden-desordenorganización, no aferrándose jamás a una estructura que haya quedado completamente obsoleta o posiblemente descontextualizada, es decir, apostar por la implementación de una cultura innovadora, (Morín, 2001). Para conseguirlo, se requiere por una parte la actualización de los planes de estudio para dotarlos de una mayor calidad y coherencia, y por otro lado transformar la formación inicial del profesorado, que pasa por la inclusión de determinadas cuestiones; lo que hemos denominado Urgencias Pedagógicas.

Principalmente, tras el análisis de los resultados de este estudio, se detecta una necesidad de formación tanto a escolares como a docentes en el uso educativo de las tecnologías como defienden Mishra y Koehler (2006) o en la curaduría de contenidos de la que habla Blanco (2013), ante la realidad de la infoxicación que se está produciendo hoy en día (Reig, 2013). Se precisa trabajar también, la difusión de una serie de valores ciudadanos como los que defienden, Ull, Martínez y Piñero (2012). Esto daría como resultado una juventud más capacitada para vivir en sociedad como aluden los resultados de Novella, Agud, Llena y Trilla (2013).

Otro de los elementos que emergen es la adquisición de competencias emocionales, que se han ido convirtiendo en imprescindibles desde la publicación de Goleman (1996). Pero, además, se considera necesaria la formación en idiomas, como recoge San Isidro (2009) y a la que el profesorado debe dar una respuesta de calidad.

Es por esta actualización constante de necesidades que Couros (2015) ya no alude a las facultades de educación como centros de formación donde se transmiten una serie de conocimientos cerrados y rígidos, si no como espacios que deben fomentar habilidades que permitan al profesorado desarrollar la función docente en las aulas presentes y futuras. Para ello, incide en prestar atención también a la creación de redes personales de aprendizaje, pues son las que nos permitirán continuar con el aprendizaje a lo largo de la vida.

Estas premisas nos advierten de la urgencia de rediseñar la formación del profesorado de manera que tenga en cuenta estas circunstancias. Existen diversas urgencias pedagógicas a las que hay que dar respuestas mediante 
una formación inicial de calidad. Debemos formar por tanto al futuro profesorado no como simples distribuidores de contenidos disciplinares, si no como personas gestoras de esos contenidos. Ofrecer una formación tanto en el uso de las TIC, como en un uso pedagógico y responsable de las mismas, haciéndoles conscientes de los diversos contextos en los que se desarrolla la educación, la gran diversidad que encontramos actualmente en las aulas supone una mayor formación para llegar de manera más eficaz a las necesidades de cada alumna y alumno, por muy diferentes que sean.

En síntesis, se podría afirmar que el nuevo contexto tecnológico está cambiando el modelo educativo hacia otros estándares de calidad ligados a competencias y habilidades, como señalan Viorica-Tori y Carmen (2012), que permitan a las personas desarrollarse en sociedad. Pero para que de verdad esto ocurra, es necesario un cambio en el sistema educativo, generar una cultura escolar innovadora que como demanda Bas (2014) se base en el pragmatismo, la contextualización, que sea sostenible y capaz de moverse de manera estratégica. Para ello, es fundamental una transformación educativa que consiga calar hasta transformar la sociedad. Esto implica de un diálogo igualitario entre los participantes de la comunidad educativa como sostiene Álvarez Álvarez (2015). En palabras de Aubert, et al. (2008) necesitamos de una Educación Dialógica gestionada mediante comunidades de aprendizaje para posibilitar ese diálogo igualitario que consiga transformar la educación y con ella la sociedad.

En el proceso de cambio que nos encontramos, seguir educando conforme a una realidad que ya no existe solo supondrá la gestación de una ciudadanía incapaz de desenvolverse en el nuevo contexto. En cambio, proporcionar una educación contextualizada que dote a las personas de las herramientas y saberes necesarios para hacer frente al mundo actual, supondrá la diferenciación clave para alcanzar la verdadera prosperidad. Con todo, sería interesante conocer la opinión del profesorado y alumnado de otros niveles educativos, así como de otros grupos de la población acerca de este tema, dado que este estudio recoge únicamente la visión de la comunidad universitaria. De esta forma, al aumentar el número de participantes sería posible desarrollar los análisis realizados en mayor profundidad y facilitar la exploración de nuevas categorías de análisis, con 
22 Valdés \& Gutiérrez-Urgencias Pedagógicas

el objetivo de continuar ahondando en el sentido de la educación y las Urgencias Pedagógicas.

\section{Referencias Bibliográficas}

Álvarez Álvarez, C. (2015). De la innovación educativa a la transformación social: teoría y práctica. Intangible Capital, 11(3), 285-292.

doi: 10.3926/ic.663

Anguita, R. (2011). El reto de la formación del profesorado para la igualdad. Revista Electrónica Interuniversitaria de Formación del Profesorado, 14(1), 43-51. Retrieved from

http://www.aufop.com/aufop/uploaded_files/articulos/1301587682.pdf

Area, M. (2010). ¿Por qué formar en competencias informacionales y digitales en la educación superior? Competencias informacionales y digitales en educación superior. Revista de Universidad y Sociedad del Conocimiento (RUSC), 7(2), 1-62. Retrieved from

http://openaccess.uoc.edu/webapps/o2/bitstream/10609/2601/2/Monografic _esp.pdf

Area, M., \& Pessoa, T. (2012). De lo sólido a lo líquido: las nuevas alfabetizaciones ante los cambios culturales de la web 2.0. Comunicar: revista científica de comunicación y educación, 38, 13-20. doi: 10.3916. C38-2011-02-01

Areekul, C., Ratana-Ubol, A., \& Kimpee, P. (2015). Model development for strengthening social capital for being a sustainable lifelong learning society. Procedia-Social and Behavioral Sciences, 191, 1613-1617. doi: 10.1016/j.sbspro.2015.04.508

Attwell, G. (2007). Personal Learning Environments-the future of eLearning? Elearning papers, 2(1), 1-8. Retrieved from https://www.researchgate.net/publication/228350341

Aubert, A., Flecha, A., García, C., Flecha, R., \& Racionero, S. (2008). Aprendizaje dialógico en la Sociedad de la Información. Barcelona: Hipatia.

Bas, E. (2014). Educar para innovar: La innovación como cultura. Juventud, proactividad, creatividad, participación y visión de futuro compartida. Revista de Estudios de Juventud, 104, 11-30. Retrieved from 
REMIE - Multidisciplinary Journal of Educational Research, 8(1)23

http://www.injuve.es/sites/default/files/Documento\%201.\%20Educar\%20pa ra\%20innovar.pdf

Bhatt, V. (2016). Information Literacy Competencies in New Era. IJRARInternational Journal of Research and Analytical Reviews, 3(4), 13-19. Retrieved from http://ijrar.com/upload_issue/ijrar_issue_326.pdf

Biesta, G. (2014). Cultivating humanity or educating the human? Two options for education in the knowledge age. Asia Pacific Education Review, 15(1), 13-19. doi: 10.1007/s12564-013-9292-7

Blanco, L. E. (2013). Gestión y curación de contenidos como herramienta para educadores y comunicadores. Temas de comunicación, 27, 107-123.

Retrieved from

http://revistasenlinea.saber.ucab.edu.ve/temas/index.php/temas/article/down load/2025/2092

Cantón, I. (2001). Nueva organización escolar en la sociedad de conocimiento. Bordón. Revista de pedagogía, 53(2), 201-214. Retrieved from https://dialnet.unirioja.es/servlet/articulo?codigo $=54697$

Cera, E. (2012). La educación emocional en los planes de estudio del grado de primaria en la comunidad andaluza. Revista Wanceulen EF digital, 9, 3744. Retrieved from http://rabida.uhu.es/dspace/bitstream/handle/10272/5658/La_educacion_em ocional_en_los_planes_de_estudio.pdf?sequence=2

Cobo, R. (coord.) (2008). Educar en la ciudadanía. Perspectivas feministas. Madrid: Catarata.

Cobo, C., \& Moravec, J. (2010). Invisible learning. Barcelona: Laboratori de Mitjans Interactius (University of Barcelona Press).

Consejo de Universidades (2010). La formación permanente y las universidades españolas. Retrieved from http://www. educacion. gob.es/dctm/eu2015/2010formacion- permanente-universidadesespanolas-060710. pdf.

Contreras Domingo, J., \& Pérez de Lara, N. (2010). Investigar la experiencia educativa. Madrid: Morata.

Couros, A. (2015). Developing Teacher Candidates in a Networked World. It's no longer enough for faculties of education to deliver static, technical courses on the methods of teaching. Retrieved from http://www.ceaace.ca/blog/alec-couros- and-katia- hildebrandt/2015/03/3/developingteacher-candidates-networked-world 


\section{Valdés \& Gutiérrez-Urgencias Pedagógicas}

Dabbagh, N. \& Kitsantas, A. (2012). Personal Learning Environments, social media, and self-regulated learning: A natural formula for connecting formal and informal learning. Internet and Higher Education, 15, 3-8. doi: 10.1016/j.iheduc.2011.06.002

De Lara, P. M. M. (2008). El conflicto en la escuela: una oportunidad de aprendizaje. Bordón. Revista de Pedagogía, 60(4), 125-136. Retrieved from https://dialnet.unirioja.es/descarga/articulo/2912307.pdf

Delors, J. (1996). La educación encierra un tesoro. Madrid: Santillana.

Fernández-Soria, J.M., \& Mayordomo, A. (2014). La finalidad política y cívica de la educación. El origen de una tradición histórica en España. Bordón. Revista de pedagogía, 66(2), 107-120. doi: 10.13042/Bordon.2014.66207

Funes, S. (2000). Resolución de conflictos en la escuela: Una herramienta para la cultura de paz y convivencia. Contextos educativos, 3, 91-106. Retrieved from https://dialnet.unirioja.es/descarga/articulo/201067.pdf

García Raga, L. \& López Martín, R. (2013). La convivencia escolar y la construcción de la ciudadanía. Balance retrospectivo y desafíos de futuro. Bordón. Revista de Pedagogía, 66(2), 93-106. doi:

10.13042/Bordon.2014.66206

García, R., Rebollo, M. A., Buzón, O., González-Piñal, R., Barragán, R., \& Ruiz, E. (2010). Actitudes del alumnado hacia la igualdad de género. Revista de Investigación Educativa, 28(1), 217-232. Retrieved from http://revistas.um.es/rie/article/view/98951

Gimeno Sacristán, J. (1982). La pedagogía por objetivos: obsesión por la eficiencia. Madrid: Morata.

Goleman, D. (1996). Inteligencia emocional. Barcelona: Kairós.

Gómez, L. (2000). Educación para la ciudadanía. Madrid: UGT-Escuela Julián Besteiro.

Gutiérrez, P., \& Becerra, Ma . T. (2014). Los Entornos Personales de Aprendizaje (PLE). Una experiencia de aprendizaje informal en la formación inicial del profesorado. RELATEC, Revista Latinoamericana de Tecnología Educativa, 13(2), 49-60. doi: 10.17398/1695-288X.13.2.49 Gutiérrez, P., \& Mikiewicz, P. (2012). Learning on the Edge. Edgeryders learning and educational experiences. Report to the Council of Europe. Brussels: Council of Europe. 
Gutiérrez Díez, E. (2015). La construcción educativa del nuevo sujeto neoliberal. Cuadernos de pedagogía, 454, 76-81. Retrieved from http://hdl.handle.net/10612/6726

Hall, R. (2009). Towards a Fusion of Formal and Informal Learning Environments: the Impact of the Read/Write Web. Electronic Journal of eLearning, 7(1), 29-40. Retrieved from www.ejel.org

Kafai, Y. B., \& Peppler, K. A. (2011). Youth, technology, and DIY:

Developing participatory competencies in creative media production.

Review of research in education, 35(1), 89-119. doi:

10.3102/0091732X10383211

Karpov, A. O. (2017). The Problem of Separating the Notions of "Knowledge" and "Information" in the Knowledge Society and its Education. ProcediaSocial and Behavioral Sciences, 237, 804-810. doi:

10.1016/j.sbspro.2017.02.152

Kartashova, A. (2015). Cultural and Historical Correlations of Ideal Education and Human Paradigm. Procedia. Social and Behavioral Sciences, 166(7), 351-355. doi: 10.1016/j.sbspro.2014.12.535

Kim, S. \& Kim, K.H. (2013). Reconsidering Education for Sustainable Society: An East Asian Perspective. Multidisciplinary Journal of Educational Research, 3(1 ), 1 -1 8. doi: 1 0.4471/remie.201 3.01

Kornienko, A. A. (2015). The concept of knowledge society in the ontology of modern society. Procedia-Social and Behavioral Sciences, 166, 378-386. doi: 10.1016/j.sbspro.2014.12.540

Laal, M., Laal, A., \& Aliramaei, A. (2014). Continuing education; lifelong learning. Procedia-Social and Behavioral Sciences, 116, 4052-4056. doi: 10.1016/j.sbspro.2014.01.889

Leite, C., \& Fernandes, P., \& Marques da Silva, S. (2013). O lugar da educação para a cidadania no sistema educativo português: perspetivas de docentes de uma escola TEIP. Educação, 36 (1), 35-43. Retrieved from http://www.redalyc.org/articulo.oa?id=84825694006

Mishra, P., \& Koehler, M. J. (2006). Technological Pedagogical Content Knowledge: A Framework for Teacher Knowledge. The Teachers College Record, 108(6), 1017-1054. doi: 10.1111/j.1467-9620.2006.00684.x

Molina Girón, L. A. (2013). ¿Cómo la escuela educa para una ciudadanía activa? Una experiencia de educación cívica ciudadana en Canadá. 


\section{Valdés \& Gutiérrez-Urgencias Pedagógicas}

Multidisciplinary Journal of Educational Research, 3(3), 296-326. doi: 10.4471/remie.2013.17

Moral, C. (2008). Aprender a pensar-aprender a aprender. Habilidades de pensamiento y aprendizaje autorregulado. Bordón. Revista de Pedagogía, 60(2), 123-138. Retrieved from

https://dialnet.unirioja.es/servlet/articulo?codigo=2717070

Morilla-García, C. (2017). The Role of Emotional Intelligence in Bilingual

Education: A Study on The Improvement of The Oral Language Skill.

Multidisciplinary Journal of Educational Research, 7(1), 27-52.

doi:10.17583/remie.2017.1840

Masini, E. B. (2013). Intergenerational responsibility and education for the future. Futures, 45, 32-37. doi: 10.1016/j.futures.2012.11.005

Morín, E. (2001). Los siete saberes necesarios para la educación del futuro.

Barcelona: Paidós.

Ngre, F., Marín, V.I., \& Pérez, A. (2013). Estrategias para la adquisición de la competencia informacional en la formación inicial de profesorado de primaria. Revista Electrónica Interuniversitaria de Formación del Profesorado, 16(2), 1-12. doi: 10.6018/reifop.16.2.180751

Novella, A.M., Agud, I., Llena, A., \& Trilla, J. (2013). El concepto de ciudadanía construido por jóvenes que vivieron experiencias de participación infantil. Bordón. Revista de Pedagogía, 65(3), 93-108. doi: $10.13042 / 23166$

Palmer, N. (2017). Cultivating global citizenship education in context. Journal of Academic Perspectives, 2017(2), 1-13. Retrieved from https://www.researchgate.net/publication/320628255

Pérez Gómez, A. I. (2000). Las finalidades de la educación. Madrid: UGTEscuela Julián Besteiro.

Ponerulappan. P., \& Thilagavathy, N. (2014). The Impact of Content Curation for Personal/Informal Learning. International Journal of Science and Research (IJSR), 5(1), 1613-1676. Retrieved from https://www.ijsr.net/archive/v5i1/NOV153170.pdf

Rahimi, E., Van den Berg, J., \& Veen, W. (2015). Facilitating student-driven constructing of learning environments using Web 2.0 personal learning environments. Computers \& Education, 81, 235-246. doi:

10.1016/j.compedu.2014.10.012 
REMIE - Multidisciplinary Journal of Educational Research, 8(1)27

Reig, D. (2013). Viajando hacia la utopía de la mano de las Tecnologías.

Boletín SCOPEO No 84. 15 de mayo de 2013. Retrieved from

http://scopeo.usal.es/viajando-hacia-la-utopía-de-la-mano-de-las-

tecnologías/

Resende, T. F. (2013). “Aprender a conhecer” na sociedade da informação: o papel específico da escola e os seus desafíos. RASE: Revista de la Asociación de Sociología de la Educación, 6(2), 289-301. Retrieved from https://dialnet.unirioja.es/servlet/articulo?codigo $=5144565$

Ricaurte, P. (2016). Pedagogies for the open knowledge society. International Journal of Educational Technology in Higher Education, 13(1), 32. doi 10.1186/s41239-016-0033-y

Rimbau, E., Delgado. A. M., \& Rifá, H. (septiembre de 2008). El reconocimiento del aprendizaje experiencial: un elemento clave en el EEES. Comunicación presentada a las $V$ Jornadas de Innovación Universitaria, Madrid.

Rodríguez, M., Orozco, M., \& Larena, R. (2011). Educación para el desarrollo, papel imprescindible de la universidad. Revista Electrónica

Interuniversitaria de Formación del Profesorado, 14(1), 125-136.

Retrieved from https://dialnet.unirioja.es/descarga/articulo/3678784.pdf Rodríguez Fuentes, A., Ayllón Blanco, M. F., Gallego Ortega, J. L., \& Gómez Pérez, I. A. (2017). The Communication Skills of Future Teachers during their Initial Training. Multidisciplinary Journal of Educational Research, 7(1), 88-118. doi:10.17583/remie.2017.2200

San Isidro, X. (2009). CLIL, un nuevo horizonte en la formación del profesorado. Latin American Journal of Content \& Language Integrated Learning, 2(1), 51-54. doi:10.5294/lacli1.2009.2.1.8

Selwyn, N. (2007). The use of computer technology in university teaching and learning: a critical perspective. Journal of computer assisted learning, 23(2), 83-94. doi: 10.1111/j.1365-2729.2006.00204.x

Sevillano, M.L. (2005). Didáctica en el siglo XXI. Ejes en el aprendizaje y enseñanza de calidad. Madrid: Mc Graw Hill.

Strauss, A., \& Corbin, J. (2002). Bases de la investigación cualitativa.

Técnicas y procedimientos para desarrollar la teoría fundamentada.

Medellín: Editorial Universidad de Antioquia.

Tien, P. D. N. (diciembre de 2013). Characteristics of Learning Societies and Learning Citizens: A Literature Review. Reference document for the 


\section{Valdés \& Gutiérrez-Urgencias Pedagógicas}

National Workshop on 'Building a Learning Society in Vietnam: From Vision to Action', Hanói, Vietnam.

Tünnermann, C. (2010). La educación permanente y su impacto en la formación superior. Revista Iberoamericana de Educación Superior, 1(1), 120-133. Retrieved from http://www.redalyc.org/articulo.oa?id=299128587011

Turturean, M. (2015). Rethinking the Role of Adults for Building the LIFELONG Learning Society. Procedia-Social and Behavioral Sciences, 180, 1215-1220. doi: 10.1016/j.sbspro.2015.02.249

Vergara, J. (coord.) (2008). Formación para la ciudadanía. Un reto de la sociedad educadora. Barcelona: Ariel.

Viorica-Torii, C., \& Carmen, A. (2012). The Role of Student-Oriented Educational Strategies in Initial Teacher Training. Procedia-Social and Behavioral Sciences, 51, 757-762. doi: 10.1016/j.sbspro.2012.08.236 Yuste, R. (2012). Una E-Evaluación innovadora como factor de mejora en la enseñanza on- line. Badajoz, Facultad de Educación, Universidad de Extremadura. Tesis doctoral.

Zambrano Farías, F. J. (2017). Sociedad del Conocimiento y las TEPs. INNOVA Research Journal, 2(10), 169-177. Retrieved from http://www.journaluidegye.com/magazine/index.php/innova/article/view/53 $4 / 475$

Víctor Valdés Sánchez es Candidato a Doctor de Educación, Universidad de Extremadura.

Prudencia Gutiérrez Esteban es Profesora Contratada Doctora en el área de Didáctica y Organización Escolar en la Universidad de Extremadura

Contact Address: Departamento de Didáctica y Organización Escolar. Facultad de Educación. Avda. de Elvas, s/n, 06006 Badajoz.

Email: vvaldess@alumnos.unex.es 